\title{
USO DO BLUE RAYON PARA EXTRAÇÃO/CONCENTRAÇÃO DE COMPOSTOS POLICÍCLICOS EM AMOSTRAS AMBIENTAIS
}

\author{
Fábio Kummrow* e Gisela de Aragão Umbuzeiro \\ Faculdade de Ciências Farmacêuticas, Universidade de São Paulo, Av. Prof. Lineu Prestes, 580, 05508-900 São Paulo - SP, Brasil \\ Companhia de Tecnologia de Saneamento Ambiental, Av. Prof. Frederico Hermann Jr., 345, 05459-900 São Paulo - SP, Brasil
}

Recebido em 18/2/05; aceito em 21/7/05; publicado na web em 16/2/06

\begin{abstract}
USE OF BLUE RAYON FOR THE EXTRACTION/CONCENTRATION OF POLYCYCLIC COMPOUNDS IN THE AQUATIC ENVIRONMENT. This is a review about the use of Blue rayon in the extraction and concentration of environmental contaminants in the aquatic environment. Blue rayon is an adsorbent composed of fibers covalently linked with copper phthalocyanine trisulphonate that has the ability to selectively adsorb polycyclic compounds. Blue rayon can be used in situ, in columns or in flasks. This method showed to be efficient in the extraction of important classes of environmental contaminants like the polycyclic aromatic hydrocarbons (PAHs), aromatic amines and phenylbenzotriazoles (PBTAs) and can be an important tool in monitoring studies for the evaluation of water quality.
\end{abstract}

Keywords: Blue rayon; polycyclic compounds; genotoxic contaminants.

\section{INTRODUÇÃO}

A poluição nos diferentes compartimentos ambientais (águas, solos e ar), que resulta como principal consequiência do processo de industrialização ou simplesmente de atividades humanas, é um dos principais problemas que o homem tem para resolver ou, pelo menos, controlar. Os contaminantes ambientais podem ter diferentes características químicas e, em uma classificação preliminar, podem ser divididos em poluentes orgânicos, inorgânicos e espécies metálicas ${ }^{1}$.

Os micropoluentes orgânicos são um dos problemas mais críticos relativos aos recursos de água para consumo humano, e à proteção dos ecossistemas aquáticos ${ }^{2}$. Neste grupo de contaminantes, os produtos químicos tóxicos ou cancerígenos têm recebido grande atenção ${ }^{3}$.

Para análise dos contaminantes presentes em ambientes aquáticos, os procedimentos de extração/concentração são muito utilizados, pois estes normalmente estão em concentrações baixas, diluídos em grandes volumes de amostra, e os métodos seletivos de extração/concentração de amostras têm se mostrado bastante úteis no direcionamento das análises químicas de identificação, bem como na descoberta de novas classes de contaminantes ambientais ${ }^{3-9}$.

Em 1983 foi descoberto que fibras de algodão ligadas covalentemente ao pigmento ftalocianina de cobre tiossulfato, denominadas Blue cotton (Figura 1), formavam um adsorvente seletivo para compostos policíclicos ${ }^{10,11}$.

O Blue rayon, similarmente ao Blue cotton, é um conjunto de fibras inertes de rayon ligadas covalentemente ao mesmo pigmento, ftalocianina de cobre tiossulfato, contendo 2-3 vezes mais ligantes (aproximadamente $30 \mu \mathrm{mol} / \mathrm{g}$ peso seco) que o Blue cotton (10 $\mu \mathrm{mol} / \mathrm{g}$ peso seco). A estrutura do Blue rayon não foi encontrada na literatura pesquisada ${ }^{12-14}$. O Blue rayon tem sido mais utilizado que o Blue cotton como adsorvente de substâncias com estrutura molecular planar e policíclica presentes em amostras de alimentos, águas e materiais biológicos ${ }^{11,15,16}$.

\footnotetext{
*e-mail: fabiok@cetesbnet.sp.gov.br
}

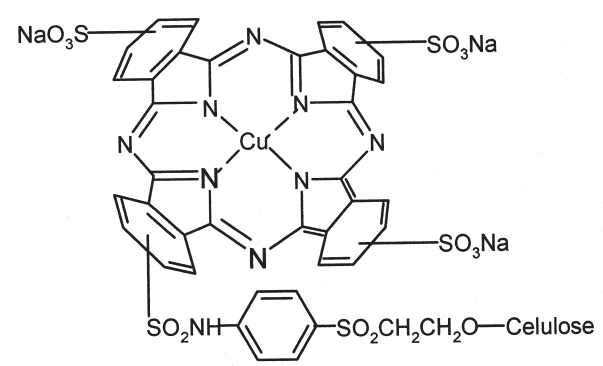

Figura 1. Estrutura do Blue cotton

Com o objetivo de obter um adsorvente com mais moléculas de ftalocianina de cobre tiossulfato e, portanto, maior número de sítios de ligação para os contaminantes ambientais, foi desenvolvida a Blue chitin. A quitina em pó é capaz de conter aproximadamente $40 \mu \mathrm{mol} / \mathrm{g}$ peso seco do pigmento. Normalmente a Blue chitin é utilizada em pequenas colunas para extração em fase só$\operatorname{lida}^{11}$.

O mecanismo pelo qual o Blue cotton, Blue rayon e a Blue chitin adsorvem os compostos policíclicos está diretamente relacionado com a característica planar deste tipo de molécula ${ }^{16}$. Uma ligação hidrofóbica 1:1 é formada com o pigmento ftalocianina de cobre tiossulfato, que tem uma grande estrutura molecular planar ${ }^{14}$. Preferencialmente são adsorvidos pelo Blue rayon os compostos com três ou mais anéis fundidos em sua molécula. Hayatsu ${ }^{16}$, em um estudo de recuperação onde compostos com um e dois anéis e alifáticos foram avaliados, observou que os mesmos apresentaram baixa ou moderada recuperação. Na Tabela 1 foram sumarizados os resultados obtidos nesse trabalho.

Para a identificação dos compostos policíclicos presentes nos extratos obtidos com o Blue rayon há uma tendência de se combinar testes de genotoxicidade com análises químicas. Usualmente testam-se os extratos orgânicos totais e suas frações através do teste conhecido como Salmonella/microssoma ou Teste de Ames ${ }^{17,18}$. As frações com maior atividade mutagênica são submetidas às análises químicas de identificação. Esta estratégia tem sido utilizada 
Tabela 1. Recuperação aproximada de compostos policíclicos e não policíclicos por Blue cotton, baseada nos gráficos da ref. 16

\begin{tabular}{|c|c|c|}
\hline Composto & $\begin{array}{l}\text { Número } \\
\text { de anéis }\end{array}$ & $\begin{array}{c}\% \text { de } \\
\text { recuperação }\end{array}$ \\
\hline $\mathrm{A} \alpha \mathrm{C}$ & $\geq 3$ & 90 \\
\hline 2-acetilaminofluoreno & $\geq 3$ & 55 \\
\hline $\mathrm{N}$-acetil-Trp-P-1 & $\geq 3$ & 95 \\
\hline $\mathrm{N}$-acetil-Trp-P-2 & $\geq 3$ & 75 \\
\hline Hidroclorito de acrivlavina & $\geq 3$ & 100 \\
\hline Actinomicina D & $\geq 3$ & 25 \\
\hline Aflatoxina $\mathrm{B}_{1}$ & $\geq 3$ & 85 \\
\hline 9-aminoacridina & $\geq 3$ & 95 \\
\hline 2-aminoantraceno & $\geq 3$ & 75 \\
\hline Bellidifolin & $\geq 3$ & 60 \\
\hline Benz[a]antraceno 5-metilenosulfato & $\geq 3$ & 50 \\
\hline Benzo[a]pireno & $\geq 3$ & 100 \\
\hline Ácido carmínico & $\geq 3$ & 45 \\
\hline Hidroclorito de clorpromazina & $\geq 3$ & 80 \\
\hline $\begin{array}{l}\text { Ácido tetrasulfônico ftlocianina } \\
\text { de cobre }\end{array}$ & $\geq 3$ & 85 \\
\hline Daunorubicina & $\geq 3$ & 100 \\
\hline $1, \mathrm{~N}^{6}$-etenoadenosina & $\geq 3$ & 0 \\
\hline Brometo de etídio & $\geq 3$ & 95 \\
\hline Etil eosina & $\geq 3$ & 95 \\
\hline Fluoreceina & $\geq 3$ & 50 \\
\hline Glu-P-1 & $\geq 3$ & 85 \\
\hline Glu-P-2 & $\geq 3$ & 65 \\
\hline Harman & $\geq 3$ & 95 \\
\hline Heme & $\geq 3$ & 85 \\
\hline IQ & $\geq 3$ & 95 \\
\hline $\mathrm{Me} A \alpha \mathrm{C}$ & $\geq 3$ & 90 \\
\hline MeIQ & $\geq 3$ & 90 \\
\hline MeIQx & $\geq 3$ & 95 \\
\hline 8-metoxipsoralen & $\geq 3$ & 60 \\
\hline Azul de metileno & $\geq 3$ & 100 \\
\hline 1-nitropireno & $\geq 3$ & 80 \\
\hline Norharman & $\geq 3$ & 90 \\
\hline Fenasina A & $\geq 3$ & 85 \\
\hline Fenasina $\mathrm{C}$ & $\geq 3$ & 70 \\
\hline Hidroclorito de quinacrina & $\geq 3$ & 100 \\
\hline Rodamina & $\geq 3$ & 100 \\
\hline Riboflavina & $\geq 3$ & 80 \\
\hline Swertianolina & $\geq 3$ & 30 \\
\hline Trp-P-1 & $\geq 3$ & 100 \\
\hline Trp-P-2 & $\geq 3$ & 100 \\
\hline Adenina & 2 & 10 \\
\hline Adenosina & 2 & 2 \\
\hline Amarant & 2 & 35 \\
\hline ATP & 2 & 0 \\
\hline Carbadox & 2 & 35 \\
\hline 6-dimetilalilaminopurina & 2 & 35 \\
\hline NADH & 2 & 0 \\
\hline Naftaleno 1-metilenosulfato & 2 & 10 \\
\hline 4-nitroquinolina 1-óxido & 2 & 10 \\
\hline PhIP & 2 & 95 \\
\hline Quercetina & 2 & 95 \\
\hline Triptofano & 2 & 5 \\
\hline 4-aminobifenil & 1 & 35 \\
\hline Furilfumarida & 1 & 25 \\
\hline Histidina & 1 & 2 \\
\hline Mitomicina & 1 & 0 \\
\hline 4-nitro- $O$-fenilenediamine & 1 & 15 \\
\hline p-nitrofenol-Na & 1 & 5 \\
\hline Fenolftaleína & 1 & 25 \\
\hline Nitrosodimetilamina & 0 & 0 \\
\hline Ácido oleico-NA & 0 & 10 \\
\hline Fecapentano-12 & 0 & 20 \\
\hline
\end{tabular}

tanto para detecção de compostos conhecidos, como para identificação de novas classes de compostos mutagênicos ${ }^{4,15,19}$.

Existem diferentes linhagens de Salmonella que podem ser utilizadas e que respondem seletivamente a classes específicas de compostos químicos. A seletividade das linhagens associada à seletividade das técnicas de extração/concentração de amostras pode levar à obtenção de extratos com um número reduzido de compostos, facilitando as análises de identificação química.

Para extração/concentração de contaminantes policíclicos presentes em ambientes aquáticos, Blue rayon e Blue cotton podem ser utilizados in situ, em colunas ou frascos, dependendo dos objetivos do estudo. A Tabela 2 apresenta um resumo desta revisão, compilando os compostos identificados em águas e efluentes de diferentes locais do mundo concentrados por Blue rayon.

\section{LAVAGEM E REUTILIZAÇÃO DAS FIBRAS DE BLUE RAYON}

O Blue rayon pode ser reutilizado ${ }^{16} \mathrm{e}$, para isso, deve ser lavado. Kummrow et al. ${ }^{20,21}$ padronizaram e otimizaram um procedimento de lavagem, e avaliaram o desempenho das fibras reutilizadas por comparação com a técnica de extração/concentração XAD-4 utilizando amostras de água enriquecidas com o 2-aminoantraceno (2AA).

Após a eluição, as fibras de Blue rayon devem permanecer em capela de segurança química para evaporação completa da solução de eluição residual. Após a secagem, devem ser transferidas para um becker contendo água ultrapura. O Blue rayon deve ser lavado com ajuda de um bastão de vidro, e este procedimento deve ser repetido quatro vezes. Após a última lavagem, o excesso de água deve ser retirado com papel filtro ${ }^{20}$.

Logo após a etapa de lavagem com água ultrapura, o Blue rayon é transferido para um erlenmeyer com solução de metanol/hidróxido de amônio 50:1(v/v), que deve ser mantido sob agitação mecânica, por $1 \mathrm{~h}$. Este procedimento deve ser repetido duas vezes. Após a última repetição, adicionar solução de metanol/hidróxido de amônio 50:1(v/v) e deixar as fibras imersas por uma noite. Após este período, substituir a solução de metanol/hidróxido de amônio 50:1(v/v) por metanol puro e manter as fibras por $1 \mathrm{~h}$, agitando-as ocasionalmente. O metanol deve ser recolhido e concentrado em evaporador rotatório para avaliação da eficiência do processo de lavagem. Os extratos podem ser submetidos às análises químicas ou ao teste de Ames dependendo do propósito do seu próximo $u^{20}{ }^{20}$.

Para verificação da eficiência de recuperação de fibras de Blue rayon já utilizadas e pela resina XAD-4, foram utilizadas amostras preparadas em $5 \mathrm{~L}$ de água ultrapura aos quais foram adicionados $100 \mu \mathrm{g}$ de 2AA (uma amina aromática com três anéis em sua molécula). Estas amostras enriquecidas foram tratadas como amostras naturais e submetidas aos processos de extração/concentração, conforme descritos por Kummrow et al. ${ }^{21}$.

Neste estudo foram avaliadas as eficiências da resina XAD-4, tanto para extração em $\mathrm{pH}$ natural quanto em $\mathrm{pH}$ ácido, e de fibras de Blue rayon com mais de 10 reutilizações, tanto com amostras de água bruta quanto tratada, Blue rayon com 7 reutilizações apenas com amostras de água bruta, Blue rayon com 5 reutilizações apenas para amostras de água tratada e Blue rayon nunca utilizado, apenas lavado. Essa recuperação foi avaliada através do teste de Ames $^{21}$.

Os resultados desta avaliação mostraram que o Blue rayon utilizado apenas para extração de amostras de água tratada apresentou uma recuperação relativamente menor. De acordo com Hayatsu ${ }^{16}$, o cloro livre presente na água tratada ataca o pigmento ftalocianina de cobre tiossulfato do Blue rayon, fato que pode ser 
Tabela 2. Compostos identificados nos extratos de Blue rayon obtidos pela técnica in situ, em colunas e frascos em diferentes locais do mundo

\begin{tabular}{|c|c|c|}
\hline Compostos identificados & Local/ambiente & Ref \\
\hline \multicolumn{3}{|c|}{ Blue rayon in situ } \\
\hline Benzo(a)pireno & Ilha Seto, Japão. Ambiente marinho & 28 \\
\hline MeIQx, Trp-P-1, Trp-P-2 e PhIP & Japão. Águas do rio Yodo & 23 \\
\hline PBTA-1 & Kyoto, Japão. Águas do rio Nishitakase & 9 \\
\hline Benzo(a)pireno & Ilha Seto, Japão. Ambiente marinho & 33 \\
\hline PBTA-2 & Kyoto, Japão. Rio Nishitakase & 25 \\
\hline Trp-P-1, IQ e A $\alpha \mathrm{C}$ & Canal de Viena. Águas do rio Danúbio, & 29 \\
\hline $\begin{array}{l}\text { Harman, 6-cloroharman, } \\
\text { 8-cloroharman e 3-cloroharman }\end{array}$ & Japão. Efluentes de estação de tratamento de esgoto doméstico de Shizuoka & 29 \\
\hline PBTA-5 е РBТA-6 & Japão. Águas dos rios Tobei, Asuwa, Nishitakase, Katsura e Uji & 5 \\
\hline PBTA-4 & Japão. Águas dos rios Nikko. e Uji & 6 \\
\hline PBTA-3, РВTA-4 е РBTA-6 & Japão. Águas dos rios Mawatari, Asuwa e Kitsune & 32 \\
\hline PBTA-7 e PBTA-8 & Japão. Águas dos rios Mawatari, Asuwa, Kitsune, Nishitakase, Katsura e Uji & 31 \\
\hline HPAs & Brasil. Águas do estuário de Santos, Brasil & 30 \\
\hline \multicolumn{3}{|c|}{ Blue rayon em colunas } \\
\hline PBTAs 1 e 2 & Japão. Águas do rio Yodo & 7 \\
\hline Trp-P-1 e Trp-P-2 & Japão. Águas de rio & 24 \\
\hline PBTAs 2, 3, 4 e 6 & Japão. Amostras de efluentes de estações de tratamento de esgotos & 36 \\
\hline \multicolumn{3}{|c|}{ Blue rayon em frascos } \\
\hline Benzo(a)pireno & Japão. Amostras de água & 38 \\
\hline
\end{tabular}

observado pela perda de cor das fibras. Com base neste resultado Kummrow et l. $^{21}$ sugerem que o Blue rayon seja reutilizado apenas 4 vezes para amostras de água tratada. O Blue rayon utilizado para extração de amostras de água não tratada pode ser reutilizado mais vezes (mais de 10 vezes) mantendo eficiência similar à da resina XAD-4. Além disso, foi possível concluir que, nas condições testadas, a resina XAD-4 e os lotes de Blue rayon apresentaram eficiências de recuperação da atividade mutagênica induzida pelo 2AA muito semelhantes ${ }^{21}$.

\section{MÉTODOS DE UTILIZAÇÃo DO BLUE RAYON}

\section{Blue rayon in situ}

Sakamoto e Hayatsu ${ }^{12}$ descreveram o método denominado Blue rayon in situ. Neste método as mechas de Blue rayon são acondicionadas em redes de nylon, com um peso no fundo, e são conectadas a uma bóia. O conjunto é colocado no ponto de amostragem e as fibras permanecem imersas por 24 h. Após este período, o conjunto é removido e as mechas de Blue rayon levadas ao laboratório para os procedimentos de preparo do extrato.

Inicialmente as mechas são lavadas com água ultrapura, para retirada do material que ficou retido sobre as fibras, não ligado às moléculas de ftalocianina de cobre tiossulfato. $\mathrm{O}$ excesso de água é retirado com papel filtro e as mechas transferidas para um erlenmeyer para serem eluídas com solução de metanol/hidróxido de amônio 50:1 v/v na proporção de $80 \mathrm{~mL}$ desta solução para cada $0,5 \mathrm{~g}$ de Blue rayon ${ }^{12}$.

São muitos os trabalhos que têm utilizado esta técnica, ou com pequenas modificações, para recuperar contaminantes orgânicos presentes em amostras ambientais, os quais serão descritos a seguir , $^{4-9,13,22-30}$.

Nos estudos realizados por Sakamoto e Hayatsu ${ }^{12}$ no rio Yodo e por Kusamran et al. ${ }^{22}$ nos rios Chao Phraya, Sumida e Ara os contaminantes presentes nos extratos não foram identificados, ape- nas sua atividade genotóxica foi avaliada através do teste de Ames ${ }^{17,18}$. Devido à seletividade do Blue rayon e a atividade mutagênica observada nos extratos obtidos por Kusamran et al. ${ }^{22}$ ter sido detectada pelo teste de Ames em condições seletivas para aminas aromáticas, foi sugerido que os compostos presentes nos extratos pertenciam a esta classe.

Otsu et al. ${ }^{13}$ utilizaram o teste de Ames e a técnica de Blue rayon in situ para avaliar a distribuição e as características dos mutágenos presentes nas águas de três rios da Coréia. Este estudo mostrou que o rio que recebia efluentes industriais apresentava alta atividade mutagênica para a linhagem TA98 na presença de ativação metabólica (> 2000 revertentes/placa), sugerindo a presença de substâncias que causam mutação do tipo deslocamento do quadro de leitura do DNA, ressaltando a importância da identificação dos compostos responsáveis por esta atividade mutagênica para se avaliar o risco ao qual a população humana está exposta ao consumir a água tratada destes mananciais.

Ohe et al. ${ }^{26}$ utilizaram a técnica de Blue rayon in situ para caracterizar a mutagenicidade de rios que atravessam áreas metropolitanas da América do Norte. Embora os contaminantes presentes nos extratos não tenham sido identificados, os autores obtiveram resultados que sugerem que a atividade mutagênica estaria relacionada a aminas aromáticas.

Umbuzeiro et al. ${ }^{4}$ verificaram a presença de compostos policíclicos aromáticos no Ribeirão dos Cristais, Brasil, utilizando a técnica de Blue rayon in situ e submetendo os extratos obtidos ao teste de Ames, com as linhagens de Salmonella tiphymurium TA98, TA100, YG1041 e YG1042. Os resultados obtidos mostraram a presença de mutágenos diretos da classe de compostos nitroaromáticos e/ou aminas aromáticas policíclicas nas águas deste ribeirão.

A técnica de Blue rayon in situ mostrou-se eficiente na recuperação das aminas aromáticas, que são formadas a partir da pirólise de proteínas, 2-amino-3,8-dimetilimidazol[4,5-f]quinoxalina (MeIQx), 3-amino-1,4-dimetil-5H-pirido[4,3-b]indol (Trp-P-1), 3amino-1-metil-5H-pirido[4,3-b]indol (Trp-P-2), 2-amino-1-metil- 
6-fenilimidazol[4,5-b]piridina (PhPI), 2-amino-3-metilimidazol[4,5-f]quinolina (IQ) (Figura 2) e 2-amino-9H-pirido[2,3-b]indol $(\mathrm{A} \alpha \mathrm{C})$ presentes em águas de rios, permitindo sua identificação por técnicas cromatográficas, bem como a detecção da atividade genotóxica desta classe de compostos ${ }^{23,25}$.<smiles>Cn1c(N)nc2c3cccnc3ccc21</smiles>

Figura 2. Estrutura do 2-amino-3-dimetilimidazol[4,5-f]quinolina (IQ)

$\mathrm{Ohe}^{23}$ identificou por cromatografia líquida de alta eficiência (CLAE) nos extratos estudados as aminas aromáticas MeIQx, Trp$\mathrm{P}-1$, Trp-P-2 e PhPI presentes nas águas do rio Yodo, no Japão. Ao submeter os extratos a teste de mutagenicidade, verificou que 5,5\% da atividade genotóxica observada era relativa à presença de MeIQx, $15,6 \%$ de Trp-P-1, 2,5\% de Trp-P-2 e 0,5\% de PhPI.

Nas águas do rio Danúbio, a utilização dessa técnica permitiu a identificação de três aminas aromáticas heterocíclicas Trp-P-1, IQ e $\mathrm{A} \alpha \mathrm{C}$, que juntas representaram $26 \%$ da atividade genotóxica destes extratos quando a amostra foi submetida ao teste de Ames com a linhagem de Salmonella typhimurium TA98, na presença de ativação metabólica ${ }^{25}$.

Uma nova classe de compostos genotóxicos, os 2-fenilbenzotriazóis (PBTAs) foi descoberta e identificada em extratos obtidos através das técnicas de Blue rayon e Blue cotton in situ em rios do Japão, que recebiam efluentes de indústrias têxteis ou que continham corantes ${ }^{5,6,8,9,31}$, tendo sido esta técnica também utilizada no monitoramento destes contaminantes ${ }^{32}$.

Os PBTAs são produtos formados a partir de dinitrofenilazo corantes, usados em processos industriais como, por ex., em unidades de tingimento. Estes podem ser formados durante a cloração de efluentes industriais em estações de tratamento e, posteriormente, lançados nos rios ${ }^{6,7}$.

Nukaya et al. ${ }^{9}$, monitorando as águas do rio Nishitakase em Kyoto através das técnicas de Blue rayon e de Blue cotton in situ, isolaram cinco compostos que somaram juntos $67 \%$ da atividade mutagênica total dos extratos. Um destes compostos, responsável por $21 \%$ da mutagenicidade encontrada, foi identificado através da técnica de cristalografia por raios-X, como sendo 2-[2(acetilamino)-4-[bis(2-metoxietil)amino]-5-metoxifenil]-5-amino7-bromo-4-cloro-2H-benzotriazol (PBTA-1) (Figura 3), um potente mutágeno.

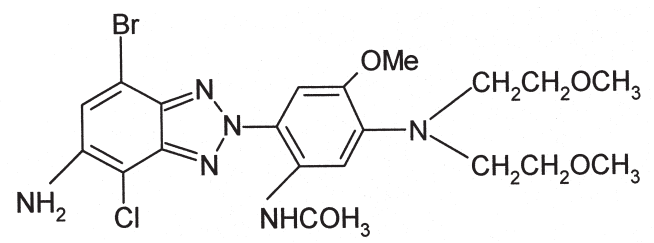

Figura 3. Estrutura do 2-[2-(acetilamino)-4-[bis(2-metoxietil)amino]-5metoxifenil]-5-amino-7-bromo-4-cloro-2H-benzotriazol (PBTA-1)

Um ano mais tarde, Oguri et al. ${ }^{8}$ identificaram outro composto presente nos mesmos extratos obtidos por Nukaya et al. ${ }^{9}$, o qual representava $17 \%$ da atividade mutagênica total dos extratos. Este foi identificado como sendo 2-[2-(acetilamino)-4-[N-(2- cianoetil)etilamino]-5-metoxifenil]-5-amino-7-bromo-4-cloro- $2 \mathrm{H}$ benzotriazol (PBTA-2), que também é um potente composto mutagênico para as linhagens de Salmonella typhimurium TA98 e YG1024 (com alta atividade da enzima acetiltransferase), em presença de ativação metabólica. Como o PBTA-1, o PBTA-2 encontrado deve ser um produto gerado a partir de corantes azóicos, formados por redução e cloração nas estações de tratamento de esgoto e efluentes ${ }^{8}$.

Ainda como continuação do trabalho de Nukaya et al. ${ }^{9}$ foi identificado um outro composto, responsável por outros $12 \%$ da atividade mutagênica total dos extratos obtidos, como sendo o 2-[2(acetilamino)-4-amino-5-metoxifenil]-5-amino-7-bromo-4-cloro2H-benzotriazol (PBTA-4) .

Watanabe et al. ${ }^{5}$ verificaram, utilizando esta técnica, que tanto o 2-[4-[bis(2-acetoxietil)amino]-2-(acetilamino)-5-metoxifenil]-5amino-7-bromo-4-cloro-2H-benzotriazol (PBTA-5) como o 2-[2(acetilamino)-4-[bis(2-hidroxietil)amino]-5-metoxifenil]-5-amino7-bromo-4-cloro-2H-benzotriazol (PBTA-6) estão presentes nos efluentes de indústrias têxteis, porém apenas o PBTA-6 foi detectado nas amostras de águas do rio que recebia os lançamentos, em níveis de 3 a $134 \mathrm{ng} / \mathrm{g}$ de Blue rayon.

Um ano mais tarde, Watanabe et al. ${ }^{31}$ detectaram e quantificaram o 2-[2-(acetilamino)-4(dietilamino)-5-metoxifenil] -5-amino-7bromo-4-cloro-2H-benzotriazol (PBTA-7) e 2-[2-(acetilamino)4(dialilamino)-5-metoxifenil]-5-amino-7-bromo-4-cloro- $2 \mathrm{H}$ benzotriazol (PBTA-8) em águas de rios do Japão. Sua mutagenicidade foi detectada através do teste de Ames, pela linhagem de Salmonella typhimurium YG1024, na presença de ativação metabólica e a contribuição do PBTA-7 para a genotoxicidade das amostras variou entre 0 e $15,7 \%$ e a do PBTA-8 entre 0 e $14,5 \%$ entre as diversas amostras analisadas.

Os PBTAs 3, 4 e 6 foram quantificados em águas de rios de Fukui, no Japão, por Watanabe et al. ${ }^{32}$, utilizando CLAE e a técnica de Blue rayon in situ. Estes PBTAs foram encontrados em $96 \%$ dos 24 extratos analisados e as quantidades encontradas para o PBTA-3 variaram entre $<0,08-58,7 \mathrm{ng} / \mathrm{g}$ de Blue rayon, para o PBTA-4 entre $<0,1-15,0 \mathrm{ng} / \mathrm{g}$ de Blue rayon e para o PBTA-6 entre $<0,07-467,9 \mathrm{ng} / \mathrm{g}$ de Blue rayon.

Em águas salgadas e salobras esta técnica tem tornado possível a identificação e quantificação relativa, por g de Blue rayon, de Hidrocarbonetos Policíclicos Aromáticos (HPAs) e alguns de seus derivados, mesmo em locais onde não era possível esta identificação por outras técnicas de extração $0^{27,30,33}$.

Após um derramamento de óleo na Baía de Galveston, Kira et $a l .{ }^{27}$ utilizaram o Blue rayon in situ para caracterizar a poluição do local e detectar a presença de compostos genotóxicos. Análises cromatográficas demostraram a adsorção no Blue rayon de HPAs mutagênicos e não mutagênicos.

Kira et $a l .{ }^{28}$ realizaram o monitoramento da atividade mutagênica e da quantidade de Benzo(a)pireno (Figura 4) em cinco áreas na região de Okayama, entre 1991 e 1992, avaliando águas e moluscos destas áreas. Nos extratos obtidos dos moluscos, a concentração de Benzo(a)pireno, relativa a $1 \mathrm{~g}$ peso seco de molusco, variou entre $0,08-3,73 \mathrm{ng} / \mathrm{g}$-equivalente e para os extratos das águas entre não detectado a $19,5 \mathrm{ng} / \mathrm{g}$ de Blue rayon.<smiles>c1ccc2c(c1)cc1ccc3cccc4ccc2c1c34</smiles>

Figura 4. Estrutura do Benzo(a)pireno 
Kummrow et al. ${ }^{30}$ identificaram e realizaram a quantificação relativa de HPAs ( $\mu \mathrm{g} / \mathrm{g}$ de Blue rayon) nas águas do estuário de Santos, no Brasil, nos extratos obtidos através da técnica de Blue rayon in situ. Nos pontos de amostragem estudados, quando utilizada a técnica de rotina baseada na extração líquido/líquido e quantificação por cromatografia líquida de alta eficiência (CLAE), os resultados eram sistematicamente abaixo do limite de detecção. Com a técnica de Blue rayon in situ foi possível detectar a presença de HPAs nessas águas e nos locais de amostragem. Onde o sedimento apresentava-se mais contaminado foram observadas maiores concentrações destes compostos nas águas.

Fukazawa et al. ${ }^{29}$ identificaram e realizaram a quantificação relativa do Harman (gerado na pirólise de proteínas) e derivados mono clorados de Harman (Figura 5) presentes no efluente final de uma estação de tratamento de efluentes domésticos em Shizuoka, Japão, utilizando a técnica de Blue rayon in situ. O Harman foi encontrado em todas as amostragens e suas concentrações variaram entre 110 a $730 \mathrm{ng} / \mathrm{g}$ de Blue rayon e os derivados mono clorados de Harman foram encontrados em concentrações que variaram entre 1 a $45 \mathrm{ng} / \mathrm{g}$ de Blue rayon, quando eram observadas concentrações altas do Harman.

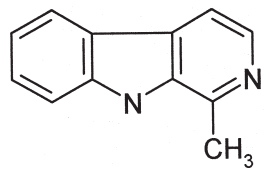

Figura 5. Estrutura do 1-metil-9H-pirido[3,4-b]indol (Harman)

Como pode ser observado nos estudos citados, a técnica in situ tem caráter qualitativo, porém a quantificação dos contaminantes presentes nos extratos pode ser feita de forma relativa, em função da massa de Blue rayon utilizada. Isso porque não é possível precisar o volume de amostra que entrou em contato com as fibras durante o período de $24 \mathrm{~h}^{34}$. Além disso, essa técnica mostrou-se adequada para caracterização da atividade genotóxica e para monitoramento da contaminação de ambientes aquáticos em relação à presença de compostos policíclicos, mesmo daqueles pouco solúveis em água ${ }^{26,27}$. A técnica in situ tem como principais vantagens a dispensa da coleta de grandes volumes de amostra, possibilidade de monitoramento em locais remotos e maior representatividade nas campanhas de amostragem por permanecer $24 \mathrm{~h}$ no ponto de amostragem $^{28,34}$.

\section{Blue rayon em colunas}

O Blue rayon pode ser utilizado também em colunas como adsorvente para extração em fase sólida. Nesta técnica as fibras previamente lavadas são empacotadas em colunas de vidro de 22/ $350 \mathrm{~mm}$, com placa porosa e torneira de teflon para cromatografia. Por elas, são passadas ${ }^{4,7,21,24}$ as amostras coletadas em campo...

Para amostras de água bruta é recomendada a proporção de $1 \mathrm{~g}$ de Blue rayon por $\mathrm{L}$ de $\operatorname{amostra}^{21,24}$, e para amostras de água tratada para abastecimento público a proporção recomendada é de 0,25 a $0,5 \mathrm{~g}$ de Blue rayon por $\mathrm{L}$ de $\operatorname{amostra}^{21,35}$.

O fluxo de passagem de amostra recomendado é $50 \mathrm{~mL}$ por $\min ^{21,35}$. Após a passagem completa das amostras, o Blue rayon é retirado da coluna e lavado com água ultrapura para retirada de sólidos suspensos e outros compostos que possam ter ficado retidos fisicamente nas fibras e não de fato adsorvidos ao pigmento ftalocianina de cobre tiossulfato ${ }^{21}$. O excesso de água deve ser retirado com papel filtro e as fibras transferidas para um erlenmeyer limpo, para eluição com metanol/hidróxido de amônio (50;1 v/v).
A eluição pode também ser realizada diretamente nas próprias colunas, como descrito por Ono et al. ${ }^{24}$, onde as fibras são lavadas com água destilada, sendo o excesso retirado com ar comprimido e a eluição realizada com metanol/hidróxido de amônio (50;1 v/v) em um fluxo de $2 \mathrm{~mL} / \mathrm{min}$. Em ambos os casos os extratos obtidos são concentrados em evaporador rotatório.

Ohe et al. ${ }^{7}$ quantificaram duas aminas aromáticas policíclicas mutagênicas, o PBTA-1 (Figura 3) e PBTA-2, no rio Yodo, utilizando o Blue rayon em colunas para concentrar as amostras e CLAE para sua identificação e quantificação. Nos mesmos extratos a mutagenicidade também foi avaliada. Nas amostras coletadas à montante do lançamento dos despejos das estações de tratamento de efluentes industriais e esgoto doméstico, as aminas não foram detectadas e os testes de mutagenicidade apresentaram resultados baixos ou negativos. Porém, nas amostras coletadas à jusante das estações de tratamento, as concentrações de PBTA-1 variaram entre $<0,01-1,91 \mathrm{ng} / \mathrm{L}$ e de PBTA-2 entre $<0,01-2,25 \mathrm{ng} / \mathrm{L}$ e os extratos apresentaram resultados positivos para os testes de mutagenicidade.

Ono et al. ${ }^{24}$ identificaram aminas heterocíclicas nas águas de rios próximos à cidade de Kyoto, onde se localizavam várias estações de tratamento de efluentes e de esgoto doméstico, utilizando esta técnica. Neste estudo, os autores identificaram por espectrometria de massa a presença de Trp-P-1 e Trp-P-2 na fração genotóxica, obtida por separação em CLAE. Concluiu-se que estas substâncias, geradas por pirólise do triptofano, eram oriundas dos efluentes das estações de tratamento de efluentes industriais e esgoto doméstico presentes na região.

Kummrow et ll. $^{21}$ aplicaram a técnica de Blue rayon em colunas em paralelo à técnica de extração com XAD-4, em amostras de águas brutas e suas respectivas águas tratadas para abastecimento público, e os extratos obtidos foram submetidos ao teste de Ames ${ }^{17,18}$. A comparação dos resultados obtidos através das diferentes técnicas de extração permitiu diferenciar a atividade mutagênica associada à presença de compostos policíclicos genotóxicos nas águas brutas, e que não foram removidos pelo processo de tratamento, da atividade dos compostos genotóxicos que são normalmente formados durante a etapa de desinfecção, durante o tratamento de águas para consumo humano (por ex. ácidos halogenados, cloral hidrato, MX, trialometanos etc.). Os estudos de Vartiainen et al. ${ }^{37} \mathrm{e}$ Kusamran et al. ${ }^{35}$ mostraram também que o Blue rayon e o Blue cotton não são capazes de recuperar os compostos genotóxicos formados durante a etapa de desinfecção por cloro utilizada no processo de tratamento de águas. Isso é explicado porque estes compostos são formados por cadeias alifáticas e o Blue rayon é seletivo para compostos policíclicos ${ }^{21,35,37}$.

Umbuzeiro et $a l .{ }^{4}$ compararam os resultados do teste de Ames $^{17,18}$ de extratos de água tratada utilizando em paralelo as técnicas de Blue rayon em colunas e XAD-4. Observaram que para a linhagem de Salmonella tiphymurium TA98 na ausência e na presença de ativação metabólica, 16 e $28 \%$ da atividade genotóxica detectada poderia ser atribuída a compostos policíclicos respectivamente e, para linhagens YG1041, nas mesmas condições, 49 e $54 \%$, respectivamente.

Utilizando o Blue rayon em colunas, Morizawa et al..$^{36}$ identificaram em amostras de efluente de estações de tratamento os PBTAs 2, 3, 4 e 6 e verificaram a contribuição destes compostos para a atividade genotóxica nos extratos obtidos. Neste estudo foi apresentado também um método para identificação simultânea de seis diferentes PBTAs e dois PBTAs não clorados.

O Blue rayon, quando utilizado em colunas, permite a quantificação dos contaminantes presentes nos extratos por volume de amostra, podendo ser utilizado como um método quantitativo para 
avaliação de compostos policíclicos presentes em ambientes aquá$\operatorname{ticos}^{34}$.

\section{Blue rayon em frascos}

A utilização do Blue rayon em frascos requer a coleta de amostras e a estas são adicionadas as mechas, de massa variável, de acordo com o objetivo do estudo e o volume de amostra. As mechas permanecem em contato com a amostra por períodos de $30 \mathrm{~min}$ a 24 h, sob agitação. Após este período o Blue rayon é retirado, lavado com água ultrapura, sendo o excesso retirado com papel filtro e, então, é realizada a eluição com metanol/hidróxido de amônio 50:1 (v/v) ${ }^{35,38}$.

A solubilidade do Benzo(a)pireno em água é muito baixa e para sua quantificação em amostras de água, Nogami et al. ${ }^{38}$ usaram o Blue rayon e extração sólido-líquido para estimar a quantidade de Benzo(a)pireno dissolvida e aquela ligada a sólidos suspensos. Do total de Benzo(a)pireno encontrado nas amostras, cerca de 60-70\% estavam ligados aos sólidos suspensos e o restante, dissolvido na amostra.

Kusamram et al. ${ }^{35}$ adicionando um g de Blue rayon a frascos contendo $4 \mathrm{~L}$ de amostras de águas tratadas para consumo humano e mantendo estes frascos sob agitação por $24 \mathrm{~h}$, também observaram que este adsorvente não é capaz de recuperar compostos genotóxicos gerados pela reação dos ácidos húmicos e fúlvicos com o cloro durante a etapa de desinfecção (ácidos halogenados, cloral hidrato, MX, etc.).

\section{ESTUDOS DE COMPARAÇÃO ENTRE O BLUE RAYON E OUTRAS TÉCNICAS DE EXTRAÇÃO}

Estudos de comparação entre o Blue rayon e outros métodos de extração/concentração foram realizados por vários autores ${ }^{4,21,35,39-41}$.

Dutka et al. ${ }^{39}$ compararam quatro métodos de concentração de amostra, incluindo o Blue rayon. Os resultados negativos para os extratos obtidos com o Blue rayon foram atribuídos à sua alta seletividade e especificidade.

$\mathrm{Ohe}^{40}$ realizou um estudo no rio Yodo, em Kyoto, utilizando teste de mutagenicidade com linhagens de Salmonella na presença e ausência de ativação metabólica, visando detectar a atividade genotóxica de compostos mutagênicos diretos e indiretos como, por ex., nitroarenos e/ou aminas aromáticas usando XAD e Blue rayon para concentrar as amostras. Os resultados obtidos mostraram-se positivos, tanto para os extratos obtidos com XAD como com o Blue rayon. Em alguns pontos de amostragem os resultados observados foram semelhantes para ambas as técnicas e em outros, a resina XAD mostrou-se mais eficiente na recuperação de compostos com atividade genotóxica. Este fato também foi atribuído à seletividade do Blue rayon para compostos policíclicos.

Sakamoto et al. $^{41}$ usaram amostras do rio Katsura, que apresentavam atividade mutagênica alta, e do rio Asahi, com atividade mutagênica moderada, para avaliar a eficiência de três técnicas de concentração: XAD-2, Blue rayon in situ e Blue chitin. Com base nos resultados obtidos, os autores concluíram que para monitoramento destes rios, as colunas de Blue chitin são mais eficientes e mais precisas que as colunas preparadas com a resina XAD-2 em avaliações quantitativas, porém, para avaliar a presença de compostos mutagênicos presentes nos rios analisados, o Blue rayon in situ é o método mais sensível e conveniente entre os três avaliados.

Kira et al..$^{33}$ concluíram que o método utilizando o Blue rayon in situ era aplicável para monitoramento de poluentes no ambiente aquático, através da similaridade da eficiência na recuperação de Benzo(a)pireno pelo Blue rayon, em comparação com um método portátil que utiliza microcolunas de extração em fase sólida.

Nos estudos que compararam a eficiência do Blue rayon em frascos $^{35}$ e em colunas ${ }^{21}$ com as resinas XAD-2 e XAD-4 para recuperação de atividade genotóxica em amostras de águas tratadas utilizando o teste de Ames foi observado que estas fibras não são capazes de recuperar compostos genotóxicos gerados durante o processo de desinfecção por cloro, normalmente presente em águas tratadas para consumo humano ${ }^{21,35}$.

\section{CONCLUSÕES}

A contaminação das águas por compostos orgânicos policíclicos, como os clássicos HPAs não substituídos, e as novas classes de contaminantes genotóxicos recém-descobertos, como o Trp-P-1, MeIQ e dos PBTAs e PBTAs não clorados, tem sido motivo de grande preocupação. Isso se deve ao fato de que tratamentos convencionais não são capazes de removê-los da água bruta, levando ao risco de exposição de populações humanas, através do consumo de águas tratadas contaminadas ou de organismos aquáticos contaminados. Como a classe dos compostos policíclicos é usualmente pouco solúvel em água, sua monitorização utilizando técnicas convencionais de extração orgânica é pouco eficiente. Desta forma o Blue rayon, especialmente in situ, pode ser uma alternativa bastante prática e adequada para monitorização da qualidade de águas naturais quanto à presença de HPAs e outros contaminantes policíclicos tóxicos.

\section{AGRADECIMENTOS}

\section{À CAPES.}

\section{REFERÊNCIAS}

1. Bruzzoniti, M. C.; Sarzanini, C.; Mentasti, E.; J. Chromatogr., A 2000, 774, 121.

2. Filipic, M.; Toman, M. J.; Water Sci. Technol. 1996, 34, 1.

3. Kataoka, H.; J. Chromatogr., A 1997, 774, 121.

4. Umbuzeiro, G. A.; Roubicek, D. A.; Rech, C. M.; Sato, M. I. Z.; Claxton, L. D.; Chemosphere 2004, 54, 1589.

5. Watanabe, T.; Nukaya, H.; Terao, Y., Takahashi; Y.; Tada, A.; Takamura, T.; Sawanishi, H.; Ohe, T.; Hirayama, T.; Sugimura, T.; Wakabayashi, K.; Mutat. Res. 2001, 498, 107.

6. Nukaya, H.; Shiozawa,T.; Tada, A.; Terao, Y.; Ohe, T.; Watanabe, T.; Asanoma, M.; Sawanishi, H.; Katsuhara, T.; Sugimura, T.; Wakabayashi, K.; Mutat. Res. 2001, 492, 73.

7. Ohe, T.; Takeuchi, N.; Watanabe, T.; Tada, A.; Nukaya, H.; Terao, Y.; Sawanishi, H.; Hirayama, T.; Sugimura, T.; Wakabayashi, K.: Environ. Health Perspect. 1999, 107, 701.

8. Oguri, A.; Shiozawa, T.; Terao, Y.; Nukaya, H.; Yamashita, J.; Ohe, T.; Sawanishi, H.; Katsuhara, T.; Sugimura, T.; Wakabayashi, K.; Chem. Res. Toxicol. 1998, 11, 1195.

9. Nukaya, H.; Yamashita, J.; Tsuji, K.; Terao, Y.; Ohe, T.; Sawanishi, H.; Katsuhara, T.; Kiyokawa, K.; Tezuca, M.; Oguri, A.; Sugimura, T.; Wakabayashi, K.; Chem. Res. Toxicol. 1997, 10, 1061.

10. Hayatsu, H.; Oka, T.; Wakata, A.; Ohara, Y.; Hayatsu, T.; Kobayashi, H.; Arimoto, S.; Mutat. Res. 1983, 119, 233.

11. Hayatsu, H.; Hayatsu, T.; Arimoto, S.; Sakamoto, H.; Anal. Biochem. 1996, $235,185$.

12. Sakamoto, H.; Hayatsu, H.; Bull. Environ. Contam. Toxicol. 1990, 44, 521.

13. Otsu, R.; Horikawa, K.; Min, B. Y.; Bull. Environ. Contam. Toxicol. 1998, 60,615 .

14. Skog, K.; J. Chromatogr., B: Biomed. Sci. Appl. 2004, 802, 39.

15. Sayato, Y.; Nakamuro, K.; Ueno, H.; Goto, R.; Mutat. Res. 1993, 300, 207.

16. Hayatsu, H.; J. Chromatogr. 1992, 597, 37.

17. Ames, B. N.; McCann, J.; Yamasaki, E.; Mutat. Res. 1975, 31, 347.

18. Umbuzeiro, G. A.; Vargas, V. M. F. Em Mutagênese Ambiental; Ribeiro, L. R.; Salvadori, D. M. F.; Marques, E. K., eds.; Ed. da ULBRA: Canoas, 2003, cap. 4.

19. Kummrow, F.; Roubicek, D. A.; Rech, C. M.; Umbuzeiro, G. A.; Rev. Bras. Ciênc. Farm. 2003, 39, 16. 
20. Kummrow, F.; Dissertação de Mestrado, Universidade de São Paulo, Brasil, 2001.

21. Kummrow, F.; Rech, C. M.; Coimbrão, C. A.; Roubicek, D. A.; Umbuzeiro, G. A.; Mutat. Res. 2003, 541, 103.

22. Kusamran, W. R.; Wakabayashi, K.; Oguri, A.; Tepsuwan, A.; Nagao, M.; Sugimura, T.; Mutat. Res. 1994, 325, 99.

23. Ohe, T.; Mutat. Res. 1997, 393, 73.

24. Ono, Y.; Somiya, I.; Oda, Y.; Water Res. 2000, 34, 890.

25. Kataoka, H.; Hayatsu, T.; Hietsch, G.; Steinkellner, H.; Nishioka, S.; Narimatsu, S.; Knasmüller, S.; Hayatsu, H.; Mutat. Res. 2000, 466, 27

26. Ohe, T.; White, P. A.; DeMarini, D. M.; Mutat. Res. 2003, 534, 101.

27. Kira, S.; Ito, T.; Hayatsu, H.; Taketa, K.; Zheng, Y.; Li, R.; Holliday, T. L.; Giam, C.S.; Bull. Environ. Contam. Toxicol. 1994, 53, 285.

28. Kira, S.; Taketa, K.; Nogami, Y.; Hayatsu, H.; Environ. Toxicol. Water Qual. 1995, 10, 167

29. Fukazawa, H.; Matsushita, H.; Terao, Y.; Mutat. Res. 2001, 491, 65.

30. Kummrow, F.; ,Coimbrão, C. A.; Franco, A.; Magalhães, D.; Umbuzeiro, G. de A.; Livro de Resumos do Congresso Brasileiro de Oceanografia, Itajaí, Brasil, 2004.
31. Watanabe, T.; Shiozawa, T.; Takahashi, Y.; Takahashi, T.; Terao, Y.; Nukaya, H.; Takamura, T.; Sawanishi, H.; Ohe, T.; Hirayama, T.; Wakabayashi, K.; Mutagenesis 2002, 17, 293.

32. Watanabe, T.; Takahashi, Y.; Takahashi, T.; Nukaya, H.; Terao, Y.; Hirayama, T.; Wakabayashi, K.; Mutat. Res. 2002, 519, 187.

33. Kira, S.; Nogami, Y.; Ito, T.; Hayatsu, H.; Mar. Environ. Res. 1998, 46, 267.

34. Ohe, T.; Watanabe, T.; Wakabayashi, K.; Mutat. Res. 2004, 567, 109.

35. Kusamran, W. R.; Tanthasri, N.; Meesiripan, N.; Tepsuwan, A.; Asian Pacific J. Canser Prev. 2003, 4, 31.

36. Morisawa, T.; Mizuno, T.; Ohe, T.; Watanabe, T.; Hirayama, T.; Nukaya, H.; Shiozawa, T.; Terao, Y.; Sawanishi, H.; Wakabayashi, K.; Mutat. Res. 2003, 534, 123.

37. Vartiainen, T.; Liimatainen, A.; Jääskeläinen, S.; Kauranen, P.; Water Res. 1987, 21, 773 .

38. Nogami, Y.; Imaeda, R.; Ito, T.; Kira, S.; Environ. Toxicol. 2000, 15, 500.

39. Dutka, B. J.; Liu, D. L.; Jurkovic, A.; McInnis, R.; Bull. Environ. Contam. Toxicol. 1993, 8, 397.

40. Ohe, T.; Water Sci. Technol. 1996, 33, 313

41. Sakamoto, H.; Ohe, T.; Hayatsu, T.; Hayatsu, H.; Mutat. Res. 1996, 371, 79. 